\title{
Full-Vectorial Optical Waveguide Mode Solvers Using Multidomain Pseudospectral Frequency-Domain (PSFD) Formulations
}

\author{
Po-Jui Chiang, Chin-Lung Wu, Chun-Hao Teng, Chu-Sheng Yang, and Hung-chun Chang, Senior Member, IEEE
}

\begin{abstract}
New full-vectorial optical waveguide eigenmode solvers using pseudospectral frequency-domain (PSFD) formulations for optical waveguides with arbitrary step-index profile are presented. Both Legendre and Chebyshev collocation methods are considered in the formulation. By applying Legendre-Lagrange or Chebyshev-Lagrange interpolating polynomials to the approximation of spatial derivatives at collocation points, the Helmholtz equations for the transverse-electric or transverse-magnetic components are converted into a matrix eigenvalue equation which is then solved for the eigenmodes by the shift inverse power method. Suitable multidomain division of the computational domain is arranged to deal with general curved interfaces of the refractive-index profile together with a curvilinear mapping technique for each subdomain so that field continuity conditions can be carefully imposed across the dielectric interfaces, which is essential in achieving high numerical accuracy. The solver is applied to the optical fiber for the assessment of its numerical performance, to the classical benchmark rib waveguide for comparing with existing high-accuracy results, and to the fused fiber structure for demonstrating its robustness in calculating the form birefingence.
\end{abstract}

Index Terms-Full-vectorial mode solver, fused fiber couplers, Helmholtz equation, optical waveguides, pseudospectral method.

\section{INTRODUCTION}

$\mathbf{C}$ ALCULATION of full-vectorial modes on optical waveguides with high accuracy has been pursued using different numerical methods, e.g., the finite difference method [1]-[3]

Manuscript received April 24, 2007; revised August 17, 2007. This work was supported in part by the National Science Council, R.O.C., under Grant NSC942215-E002-022 and Grant NSC95-2221-E-002-380, in part by the Excellent Research Projects of National Taiwan University under Grant 95R0062-AE00-06, and in part by the Ministry of Education, R.O.C., under "The Aim of Top University Plan" Grant.

P.-J. Chiang was with the Graduate Institute of Electro-Optical Engineering, National Taiwan University, Taipei, Taiwan 106-17, R.O.C. He is now with I-Shou University, Kaohsiung, Taiwan 840, R.O.C. (e-mail: d90941006@ntu. edu.tw).

C.-L. Wu was with the Graduate Institute of Communication Engineering, National Taiwan University, Taipei, Taiwan 106-17, R.O.C. He is now with Himax Analogic, Inc., Taipei, Taiwan 100, R.O.C., (e-mail: rex_wu@himaxanalogic.com.tw).

C.-H. Teng was with the Graduate Institute of Electro-Optical Engineering, National Taiwan University, Taipei, Taiwan 106-17, R.O.C. He is now with the Department of Mathematics, National Cheng Kung University, Tainan, Taiwan 701, R.O.C. (e-mail: tengch@mail.ncku.edu.tw).

C.-S. Yang was with the Department of Electrical Engineering, National Taiwan University, Taipei, Taiwan 106-17, R.O.C. He is now with the Department of Electrical Engineering and Computer Science, University of Michigan, Ann Arbor, MI 48109-2122 USA (e-mail: csyang@umich.edu).

H.-C. Chang is with the Department of Electrical Engineering, the Graduate Institute of Photonics and Optoelectronics, and the Graduate Institute of Communication Engineering, National Taiwan University, Taipei, Taiwan 106-17, R.O.C. (e-mail: hcchang@cc.ee.ntu.edu.tw).

Color versions of one or more of the figures in this paper are available online at http://ieeexplore.ieee.org.

Digital Object Identifier 10.1109/JQE.2007.910454 and the finite element method [4]. Such effort is important in the design of certain waveguide structures and devices, for example, in the analysis of form birefringence of the fiber coupler structure [3]. We have recently presented a novel method with remarkably high accuracy, the pseudospectral mode solver [5] which is based on multidomain pseudospectral methods. The multidomain advantage also helps in proper fulfillment of dielectric interface conditions, which is essential in achieving high numerical accuracy. In this paper, we discuss in detail the formulation of the pseudospectral mode solver and more numerical examples are shown.

The pseudospectral method is a well-known technique in fluid dynamics problems [6]. It has in recent years attracted more attention in the area of computational electromagnetics as an alternative solution technique owing to its high-order accuracy and fast convergence behavior over traditional methods. Its applications to electromagnetic analysis in time domain [7]-[10] and in frequency domain [11] have both been reported. Although the theory of pseudospectral method has been well applied to time-domain problems, the application to frequency-domain problems has received relatively less attention in electromagnetic problems. In contrast to pseudospectral time-domain (PSTD) techniques for time-domain problems, the so-called pseudospectral frequency-domain (PSFD) method was proposed in [11] for solving the nonhomogeneous (nonzero-source) Helmholtz equation in a simple rectangular-shape problem divided into two subdomains. In this paper, we establish waveguide mode solving schemes in frequency domain by employing the multidomain Legendre or Chebyshev collocation method, and will term the proposed method the PSFD mode solver (PSFD-MS). The curvilinear mapping technique [12] is utilized in the multidomain approach to facilitate the analysis of general waveguide structures having curved dielectric interfaces. To obtain high-accuracy full-vectorial modal solutions for dielectric waveguides, proper fulfillment of field continuity conditions across dielectric interfaces is an essential issue in whichever analysis scheme employed. Such proper treatment of continuity conditions will be carefully managed in our multidomain formulation. The Chebyshev and Legendre polynomials are appropriate for different circumstances depending on whether integral or derivative formula will be used. A matrix eigenvalue equation is finally obtained in the formulation and the eigenmodes can be readily solved by existing mathematical techniques. One recent work by Huang et al. [13] employed the multidomain pseudospectral method in the modal analysis of dielectric 


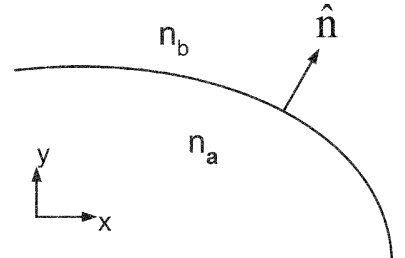

Fig. 1. Boundary between two homogeneous regions with refractive indexes $n_{a}$ and $n_{b}$ in the waveguide cross-section. $\hat{n}$ is a unit vector normal to the boundary.

waveguides with step-index profiles. However, their formulations were based on rectangular-shaped subdomains and only applied to waveguides with horizontal and vertical dielectric interfaces. Moreover, they chose to use Chebyshev polynomials in the finite subdomains and Laguerre-Gauss functions with a scaling factor in the semi-infinite regions. The optimum scaling factor for a given degree of the function, however, needs to be decided with additional efforts. In our PSFD formulation, only one kind of polynomials (Chebyshev and Legendre) is utilized, leading straightforwardly to the eigenvalue equation.

We have recently applied the similar multidomain pseudospectral method to the analysis of 2-D photonic-crystal band diagrams [14], in which the Helmholtz equation for one field component within the unit cell was solved along with the required periodic boundary conditions. For the problem in this paper, since full-vectorial modes are concerned, the eigenvectors to be solved contain two transverse field components and the formulation is a little more involved compared with the scalar problem of [14].

The rest of this paper is outlined as follows. The physical equations are described in Section II along with the required Dirichlet and Neumann type boundary conditions across the dielectric interfaces. Section III briefly discusses the Legendre and Chebyshev spectral methods. The formulation of the PSFD-MS is presented in Section IV. Numerical examples including the optical fiber, the rib waveguide, and the fused fiber structure are given in Section $\mathrm{V}$ to demonstrate the remarkable performance of the proposed scheme. The conclusion is drawn in Section VI.

\section{Physical EQuations}

Consider an isotropic-material optical waveguide of arbitrary shape with piece-wise uniform refractive index $n$ and magnetic permeability $\mu_{0}$. Fig. 1 shows a boundary of arbitrary shape between two homogeneous regions with $n=n_{a}$ and $n=n_{b}$, respectively, in the cross section of the waveguide. In this paper, we particularly pay our attention to the simple step-index structure to demonstrate the PSFD-MS's superior accuracy for optical waveguides with dramatic index change across the material interface. For an optical waveguide uniform along the $z$ axis, the magnetic field vector of a mode propagating in the $z$ direction can be expressed as $\vec{H}(x, y) \exp (-j \beta z)$, where $\beta$ is the propagation constant, which satisfies

$$
\left(\nabla^{2}+k_{0}^{2} n^{2}\right)[\vec{H}(x, y) \exp (-j \beta z)]=0
$$

where $\nabla$ is the del operator, $k_{0}=\omega / c$ is the free-space wave number, $\omega$ is the angular frequency, and $c$ is the velocity of light in vacuum. Equation (1) can be reduced to

$$
\left(\frac{\partial^{2}}{\partial x^{2}}+\frac{\partial^{2}}{\partial y^{2}}+k_{0}^{2} n^{2}\right) \vec{H}(x, y)=\beta^{2} \vec{H}(x, y) .
$$

Utilizing the pseudospectral Legendre or Chebyshev method which will be introduced in Sections III and IV, (2) will finally yield a $\beta$-formulation matrix eigenvalue equation of the form

$$
\overline{\bar{A}} \bar{H}=\beta^{2} \bar{H}
$$

where

$$
\bar{H}=\left[\bar{H}_{x}^{T}, \bar{H}_{y}^{T}\right]=\left[\begin{array}{l}
\bar{H}_{x} \\
\bar{H}_{y}
\end{array}\right]
$$

where the superscript $T$ denotes transpose, $\bar{H}_{x}$ and $\bar{H}_{y}$ are vectors composed of $H_{x}$ and $H_{y}$ values, respectively, at Legendre or Chebyshev collocation points, $H_{x}$ and $H_{y}$ are the $x$ - and $y$-components, respectively, of the magnetic field, and $\overline{\bar{A}}$ is the operator matrix which will be deduced in Section IV. The eigenvalues of (3) can be solved by applying the shift inverse power method (SIPM).

The dielectric waveguides are assumed to be non-magnetic and lossless. Referring to regions $a$ and $b$ in Fig. 1 where $\hat{n}=$ $n_{x} \hat{x}+n_{y} \hat{y}$ is a normal unit vector at the interface, the boundary conditions and Maxwell's equations lead to the Dirichlet type boundary conditions (DBCs)

$$
\begin{aligned}
H_{x}^{a} & =H_{x}^{b} \\
H_{y}^{a} & =H_{y}^{b}
\end{aligned}
$$

and the Neumann type boundary conditions

$$
\begin{aligned}
n_{x} \frac{\partial H_{x}^{a}}{\partial x}+n_{y} \frac{\partial H_{x}^{a}}{\partial y}= & n_{x}\left[-\frac{\partial H_{y}^{a}}{\partial y}+\frac{\partial H_{x}^{b}}{\partial x}+\frac{\partial H_{y}^{b}}{\partial y}\right] \\
& +n_{y}\left[\frac{\partial H_{y}^{a}}{\partial x}-\left(\frac{n_{a}}{n_{b}}\right)^{2}\left(\frac{\partial H_{y}^{b}}{\partial x}-\frac{\partial H_{x}^{b}}{\partial y}\right)\right]
\end{aligned}
$$

$$
\begin{aligned}
n_{x} \frac{\partial H_{y}^{a}}{\partial x}+n_{y} \frac{\partial H_{y}^{a}}{\partial y}= & n_{y}\left[-\frac{\partial H_{x}^{a}}{\partial x}+\frac{\partial H_{x}^{b}}{\partial x}+\frac{\partial H_{y}^{b}}{\partial y}\right] \\
& +n_{x}\left[\frac{\partial H_{x}^{a}}{\partial y}+\left(\frac{n_{a}}{n_{b}}\right)^{2}\left(\frac{\partial H_{y}^{b}}{\partial x}-\frac{\partial H_{x}^{b}}{\partial y}\right)\right]
\end{aligned}
$$

Note that we have employed the $H_{x}-H_{y}$ formulation, and (4), (5), and (6) are in terms of $H_{x}$ and $H_{y}$. In our numerical scheme, between the adjacent regions, the DBCs and NBCs are imposed across the interface to guarantee numerical stability.

\section{LegEndRE AND CHEByshev Spectral Methods}

We will formulate the multidomain PSFD-MS using the Legendre spectral method and the Chebyshev spectral method [6]. 
We describe the basic ideas of these two collocation methods in this section and show their 2-D applications.

\section{A. Legendre Spectral Method}

The Legendre polynomial $P_{N}(x)$ of order $N$ is defined as

$$
P_{N}(x)=\frac{1}{2^{N} N !} \frac{d^{N}}{d x^{N}}\left(x^{2}-1\right)^{N}
$$

where $|x| \leq 1$ and the collocation points are given as the Legendre-Gauss-Lobatto points, defined as the roots of the polynomial $\left(1-x^{2}\right) P_{N}^{\prime}(x)$, where the prime means derivative. Although there exists no analytical formula for these roots which can only be obtained numerically, the utilization of Gauss-Lobatto points can take advantage of the Gauss-Lobatto quadrature formula to significantly ameliorate accuracy in related integrals over traditional numerical integrations. If $f(x)$ is a polynomial of degree $2 N-1$, we have the formula

$$
\sum_{i=0}^{N} f\left(x_{i}\right) \omega_{i}=\int_{-1}^{1} f(\xi) d \xi
$$

where $x_{i}$ 's are the Legendre-Gauss-Lobatto collocation points and $\omega_{i}$ 's are the Gauss-Lobatto weights given as

$$
\begin{aligned}
& \omega_{i}=-\frac{2}{N+1} \frac{1}{P_{N}\left(x_{i}\right) P_{N-1}^{\prime}\left(x_{i}\right)}, \quad 1 \leq i \leq N-1 \\
& \omega_{0}=\omega_{N}=\frac{2}{N(N+1)} .
\end{aligned}
$$

In addition, the Legendre collocation method provides a means to approximating the function $f(x)$ by global Legendre-Lagrange interpolating polynomials of degree $N$

$$
f(x) \approx \sum_{i=0}^{N} f\left(x_{i}\right) g_{i}(x)
$$

where the interpolating Legendre-Lagrange polynomials are given as

$$
g_{i}(x)=-\frac{\left(1-x^{2}\right) P_{N}^{\prime}(x)}{N(N+1)\left(x-x_{i}\right) P_{N}\left(x_{i}\right)} .
$$

Then, with the interpolation of (10), the spatial derivatives of $f(x)$ at a collocation point $x_{i}$ can be computed by a matrix operator with element entries $D_{i j}=g_{j}^{\prime}\left(x_{i}\right)$, i.e.,

$$
\frac{d f\left(x_{i}\right)}{d x} \approx \sum_{j=0}^{N} g_{j}^{\prime}\left(x_{i}\right) f\left(x_{j}\right)=\sum_{j=0}^{N} D_{i j} f\left(x_{j}\right)
$$

where the explicit expression for $D_{i j}$ is given in [6] as

$$
D_{i j}= \begin{cases}\frac{-N(N+1)}{4}, & i=j=0 \\ \frac{P_{N}\left(x_{i}\right)}{P_{N}\left(x_{j}\right)} \frac{1}{x_{i}-x_{j}}, & i \neq j \\ 0, & 1 \leq i=j \leq N-1 \\ \frac{N(N+1)}{4}, & i=j=N .\end{cases}
$$

\section{B. Chebyshev Spectral Method}

The Chebyshev polynomial $T_{N}(x)$ of degree $N$ is defined as

$$
T_{N}(x)=\cos \left(N \cos ^{-1} x\right)
$$

where $|x| \leq 1$, and the collocation points are given by the Chebyshev-Gauss-Lobatto points, defined as the roots of the polynomial $\left(1-x^{2}\right) T_{N}^{\prime}(x)$. One of the merits to employ Chebyshev polynomials is the existence of the analytical formula for their collocation points, given by

$$
x_{i}=\cos \left(\frac{i \pi}{N}\right), \quad i=0,1,2, \ldots, N .
$$

Similarly, the Chebyshev collocation method provides a means to approximating the function $f(x)$ by global Chebyshev-Lagrange interpolating polynomials of degree $N$, expressed by the same (10) where $g_{i}(x)$ now denotes the interpolating Chebyshev-Lagrange polynomials given by

$$
g_{i}(x)=\frac{\left(1-x^{2}\right) T_{N}^{\prime}(x)(-1)^{i+1}}{c_{i} N^{2}\left(x-x_{i}\right)}
$$

with $c_{0}=c_{N}=2$ and $c_{i}=1$ for $1 \leq i \leq N-1$. And the spatial derivatives of $f(x)$ at a collocation point $x_{i}$ can be approximated by the same (10) with the explicit expression for $D_{i j}$ given as in [6] as

$$
D_{i j}= \begin{cases}\frac{c_{i}}{c_{j}} \frac{(-1)^{i+j}}{x_{i}-x_{j}}, & i \neq j \\ \frac{-x_{i}}{2\left(1-x_{i}^{2}\right)}, & 1 \leq i=j \leq N-1 \\ \frac{2 N^{2}+1}{6}, & i=j=0 \\ -\frac{2 N^{2}+1}{6}, & i=j=N .\end{cases}
$$

\section{2-D Formulas}

The general waveguide analysis involves 2-D functions. A 2-D function, $f(x, y)$, can be approximated as

$$
f(x, y) \approx \sum_{i=0}^{M} \sum_{j=0}^{N} f\left(x_{i}, y_{j}\right) g_{i}(x) g_{j}(y)
$$

where $y_{i}$ has been introduced as either the Legendre-Gauss-Lobatto grid or the Chebyshev-Gauss-Lobatto grid. Such approximation has the advantage that the derivatives of $f(x, y)$ can be calculated using the 1-D formula (12), and thus the approximate expressions of the spatial derivatives, $\partial f(x, y) / \partial x$ and $\partial f(x, y) / \partial y$, at the 2-D collocation points arranged in a rectangular domain can be written in the following matrix multiplication form:

$$
\begin{aligned}
& {\left[\begin{array}{cccc}
\frac{\partial f\left(x_{0}, y_{0}\right)}{\partial x} & \frac{\partial f\left(x_{0}, y_{1}\right)}{\partial x} & \ldots & \frac{\partial f\left(x_{0}, y_{N}\right)}{\partial x} \\
\frac{\partial f\left(x_{1}, y_{0}\right)}{\partial x} & \frac{\partial f\left(x_{1}, y_{1}\right)}{\partial x} & \ldots & \frac{\partial f\left(x_{1}, y_{N}\right)}{\partial x} \\
\vdots & \vdots & \ldots & \vdots \\
\frac{\partial f\left(x_{M}, y_{0}\right)}{\partial x} & \frac{\partial f\left(x_{M}, y_{1}\right)}{\partial x} & \ldots & \frac{\partial f\left(x_{M}, y_{N}\right)}{\partial x}
\end{array}\right]} \\
& =\frac{\partial \overline{\bar{f}}_{\text {rect }}}{\partial x}=\overline{\bar{D}}_{(M+1) \times(M+1)} \overline{\bar{f}}_{\text {rect }}
\end{aligned}
$$




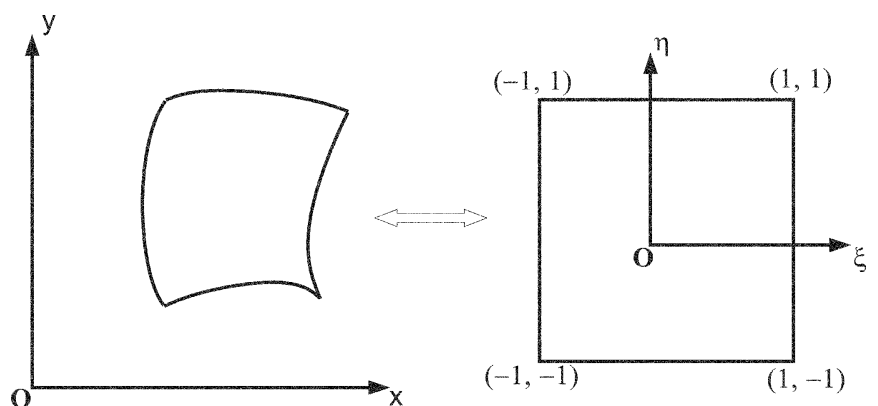

Fig. 2. Domain mapping between a region of curvilinear quadrilateral shape in the Cartesian coordinates $(x, y)$ and a square region $[-1,1] \times[-1,1]$ in the general curvilinear coordinates $(\xi, \eta)$.

$$
\begin{aligned}
& {\left[\begin{array}{cccc}
\frac{\partial f\left(x_{0}, y_{0}\right)}{\partial y} & \frac{\partial f\left(x_{0}, y_{1}\right)}{\partial y} & \ldots & \frac{\partial f\left(x_{0}, y_{N}\right)}{\partial y} \\
\frac{\partial f\left(x_{1}, y_{0}\right)}{\partial y} & \frac{\partial f\left(x_{1}, y_{1}\right)}{\partial y} & \ldots & \frac{\partial f\left(x_{1}, y_{N}\right)}{\partial y} \\
\vdots & \vdots & \ldots & \vdots \\
\frac{\partial f\left(x_{M}, y_{0}\right)}{\partial y} & \frac{\partial f\left(x_{M}, y_{1}\right)}{\partial y} & \ldots & \frac{\partial f\left(x_{M}, y_{N}\right)}{\partial y}
\end{array}\right]} \\
& =\frac{\partial \overline{\bar{f}}}{\partial y}=\bar{f}_{\text {rect }} \overline{\bar{D}}_{(N+1) \times(N+1)}^{T}
\end{aligned}
$$

where $f_{\text {rect }}$ is an $(M+1) \times(N+1)$ matrix with entries $f\left(x_{i}, y_{j}\right), i=0,1,2, \ldots, M$ and $j=0,1,2, \ldots, N$, corresponding to collocation points in rectangular arrangement, and $\overline{\bar{D}}_{(M+1) \times(M+1)}$ is an $(M+1) \times(M+1)$ matrix with entries $D_{i j}$ as defined by (13) or (17) but with $N$ replaced by $M$. Equations (19) have been given in [14] and we repeat them here for the completeness of the present formulation. Until now, the expressions of spatial derivatives in terms of matrix products are restricted to rectangular grids, which is not general enough to deal with curved boundaries. In the next section, the generalization using a curvilinear representation will be described.

\section{Multidomain PseodosPeCtRal SCHEME}

A multidomain formulation will be established in this section. The numerical scheme described above on rectangular grids will be extended to the analysis of structures with curved boundaries through the modified differential matrices. The curvilinear representation of the Helmholtz equation will be derived first and the formulation of the PSFD-MS will then be presented. Again, (20)-(22) have been presented in [14] and we describe them here for easiness of reference in later discussion.

\section{A. Curvilinear Representation of Helmholtz Equation}

The whole computational domain is divided into a suitable series of nonoverlapping curvilinear quadrilaterals by considering the profile of the waveguide cross section and material distribution. Each of these curvilinear quadrilateral regions in Cartesian $(x, y)$ coordinates is then mapped onto a square one $[-1,1] \times[-1,1]$ in curvilinear $(\xi, \eta)$ coordinates using the transfinite blending function presented in [12], as illustrated in Fig. 2, under the transformation

$$
\xi=\xi(x, y), \quad \eta=\eta(x, y) .
$$

The spatial derivatives $\partial f(x, y) / \partial x$ and $\partial f(x, y) / \partial y$ in a subdomain with curved boundaries can thus be calculated by the formulae defined on this unit element. We employ the differential matrices $\left[D_{i j}\right]$ described in Section III based on the Legendre or Chebyshev polynomial as (13) or (17) and take the degrees in $\xi$ - and $\eta$-directions in the new $(\xi, \eta)$ coordinates to be equal to $M$ and $N$, respectively. By the chain rule, the modified differential matrices are expressed as

$$
\begin{aligned}
& \frac{\partial \xi(x, y)}{\partial x} \frac{\partial}{\partial \xi} \approx \overline{\bar{D}}_{\xi x} \\
& =\left[\begin{array}{cccc}
\xi_{00}^{x} D_{00} & \xi_{01}^{x} D_{01} & \cdots & \xi_{0 M}^{x} D_{0 M} \\
\xi_{10}^{x} D_{10} & \xi_{11}^{x} D_{11} & \cdots & \xi_{1 M}^{x} D_{1 M} \\
\vdots & \vdots & \cdots & \vdots \\
\xi_{M 0}^{x} D_{M 0} & \xi_{M 1}^{x} D_{M 1} & \cdots & \xi_{M M}^{x} D_{M M}
\end{array}\right]_{(M+1) \times(M+1)}
\end{aligned}
$$

$$
\begin{aligned}
& \frac{\partial \eta(x, y)}{\partial x} \frac{\partial}{\partial \eta} \approx \overline{\bar{D}}_{\eta x} \\
& =\left[\begin{array}{cccc}
\eta_{00}^{x} D_{00} & \eta_{01}^{x} D_{10} & \cdots & \eta_{0 N}^{x} D_{N 0} \\
\eta_{10}^{x} D_{01} & \eta_{11}^{x} D_{11} & \cdots & \eta_{1 N}^{x} D_{N 1} \\
\vdots & \vdots & \cdots & \vdots \\
\eta_{N 0}^{x} D_{0 N} & \eta_{N 1}^{x} D_{1 N} & \cdots & \eta_{N N}^{x} D_{N N}
\end{array}\right]_{(N+1) \times(N+1)}
\end{aligned}
$$

$$
\begin{aligned}
& \frac{\partial \xi(x, y)}{\partial y} \frac{\partial}{\partial \xi} \approx \overline{\bar{D}}_{\xi y} \\
& =\left[\begin{array}{cccc}
\xi_{00}^{y} D_{00} & \xi_{01}^{y} D_{01} & \cdots & \xi_{0 M}^{y} D_{0 M} \\
\xi_{10}^{y} D_{10} & \xi_{11}^{y} D_{11} & \cdots & \xi_{1 M}^{y} D_{1 M} \\
\vdots & \vdots & \cdots & \vdots \\
\xi_{M 0}^{y} D_{M 0} & \xi_{M 1}^{y} D_{M 1} & \cdots & \xi_{M M}^{y} D_{M M}
\end{array}\right]_{(M+1) \times(M+1)}
\end{aligned}
$$

$$
\begin{aligned}
& \frac{\partial \eta(x, y)}{\partial y} \frac{\partial}{\partial \eta} \approx \overline{\bar{D}}_{\eta y} \\
& =\left[\begin{array}{cccc}
\eta_{00}^{y} D_{00} & \eta_{01}^{y} D_{10} & \cdots & \eta_{0 N}^{y} D_{N 0} \\
\eta_{10}^{y} D_{01} & \eta_{11}^{y} D_{11} & \cdots & \eta_{1 N}^{y} D_{N 1} \\
\vdots & \vdots & \cdots & \vdots \\
\eta_{N 0}^{y} D_{0 N} & \eta_{N 1}^{y} D_{1 N} & \cdots & \eta_{N N}^{y} D_{N N}
\end{array}\right]_{(N+1) \times(N+1)}
\end{aligned}
$$

where

$$
\begin{array}{ll}
\xi_{i j}^{x}=\frac{\partial \xi\left(x_{i}, y_{j}\right)}{\partial x}, & \eta_{i j}^{x}=\frac{\partial \eta\left(x_{i}, y_{j}\right)}{\partial x} \\
\xi_{i j}^{y}=\frac{\partial \xi\left(x_{i}, y_{j}\right)}{\partial y}, & \eta_{i j}^{y}=\frac{\partial \eta\left(x_{i}, y_{j}\right)}{\partial y}
\end{array}
$$

and $x_{i}$ and $y_{j}$ are discrete grid points corresponding to Legendre or Chebyshev collocation points in the real $(x, y)$ coordinates. Please note that when referring to (13) or (17) for the values of $D_{i j}$ 's in (21), the $x$ coordinate in (13) and (17) would become $\xi$ or $\eta$ coordinate. We can thus write the approximations for the derivatives of $f(x, y)$ in a single subdomain in terms of the operators defined in the $(\xi, \eta)$ coordinates as

$$
\begin{aligned}
& \frac{\partial \overline{\bar{f}}_{\text {cur }}}{\partial x}=\overline{\bar{f}}_{x} \overline{\bar{f}}_{\text {cur }}=\overline{\bar{D}}_{\xi x} \overline{\bar{f}}_{\text {cur }}+\overline{\bar{f}}_{\text {cur }} \overline{\bar{D}}_{\eta x} \\
& \frac{\partial \overline{\bar{f}}}{\partial y}=\overline{\bar{D}}_{y} \overline{\bar{f}}_{\text {cur }}=\overline{\bar{D}}_{\xi y} \overline{\bar{f}}_{\text {cur }}+\overline{\bar{f}}_{\text {cur }} \overline{\bar{D}}_{\eta y} .
\end{aligned}
$$

Please note that the matrix $\overline{\bar{f}}_{\text {cur }}$ is in general different from $\overline{\bar{f}}_{\text {rect }}$ in (19) in that the latter was defined on rectangular grids while 
the former is on modified locations of grids deformed according to the shape of the subdomain, and thus the operator matrices $\overline{\bar{D}}_{x}$ and $\overline{\bar{D}}_{y}$ are different from the $\overline{\bar{D}}$ matrices in (19). We then have the discrete form of (2) in a single subdomain via (22) as

$$
\overline{\bar{D}}_{x}\left(\overline{\bar{D}}_{x} \overline{\bar{H}}\right)+\overline{\bar{D}}_{y}\left(\overline{\bar{D}}_{y} \overline{\bar{H}}\right)+k_{0}^{2} n^{2} \overline{\bar{H}}=\beta^{2} \overline{\bar{H}}
$$

where the matrix $\overline{\bar{H}}=\overline{\bar{H}}_{x}$ or $\overline{\bar{H}}_{y}$, and $\overline{\bar{H}}_{x}\left(\overline{\bar{H}}_{y}\right)$ has entries that are the values of the 2-D field distribution $\overline{\bar{H}}_{x}\left(\overline{\bar{H}}_{y}\right)$ at deformed locations of grids. Note that $\overline{\bar{H}}$ is different from $\bar{H}$ in (3) which is a vector. We will convert (23) into the form of a resolvable matrix eigenvalue equation in the following subsection.

\section{B. Formulation of the PSFD-MS}

We shall describe how to relocate the matrix elements (while retaining the same values) in (23) to convert it into a resolvable form (3), i.e., $\overline{\bar{A}} \bar{H}=\beta^{2} \bar{H}$. We discuss the single subdomain setting first and then generalize to the multidomain environment.

We rearrange the $(M+1) \times(N+1)$ matrix $\overline{\bar{H}}_{i}(i=x, y)$ in (23) into the $(M+1)(N+1)$ vector column $\bar{H}_{i}$ as in (3) as

$$
\begin{gathered}
\overline{\bar{H}}_{i}=\left[\begin{array}{cccc}
H_{i 00} & H_{i 01} & \cdots & H_{i 0 N} \\
H_{i 10} & H_{i 11} & \cdots & H_{i 1 N} \\
\vdots & \vdots & \cdots & \vdots \\
H_{i M 0} & H_{i M 1} & \cdots & H_{i M N}
\end{array}\right]_{(M+1) \times(N+1)} \\
\Longrightarrow \bar{H}_{i}=\left[\begin{array}{c}
H_{i 00} \\
H_{i 10} \\
\vdots \\
H_{i M 0} \\
H_{i 01} \\
\vdots \\
H_{i M N}
\end{array}\right]_{[(M+1)(N+1)] \times 1}
\end{gathered}
$$

and then combine $\bar{H}_{x}$ and $\bar{H}_{y}$ into $\bar{H}$ in (3)

$$
\bar{H}=\left[\begin{array}{c}
\bar{H}_{x} \\
\bar{H}_{y}
\end{array}\right]_{[2(M+1)(N+1)] \times 1}=\left[\begin{array}{c}
H_{x 00} \\
H_{x 01} \\
\vdots \\
H_{x M N} \\
H_{y 00} \\
H_{y 01} \\
\vdots \\
H_{y M N}
\end{array}\right]_{[2(M+1)(N+1)] \times 1 .}
$$

The operator matrix $\overline{\bar{A}}$ in (3) can be expressed in terms of the operator matrices in (23) with some modification, shown in (26) at the bottom of the page, where $\overline{\bar{I}}$ is the identity matrix, $k=$

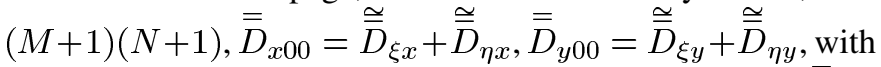
the equation shown at the bottom of the next page, where $\overline{\bar{D}}_{\xi x}$, $\overline{\bar{D}}_{\xi y}, D_{\eta x(i, j)}=\eta_{i j}^{x} D_{i j}(i=0,1, \ldots, N, j=0,1, \ldots, N)$ and $D_{\eta y(i, j)}=\eta_{i j}^{y} D_{i j}(i=0,1, \ldots, N, j=0,1, \ldots, N)$ have been given in (21), and $\operatorname{tr}[a]$ is a diagonal matrix of order $M+1$ with all diagonal elements being the constant $a$.

In the generalization to the multidomain situation, we assume that the computational domain is divided unto $p$ subdomains and the $\beta$-formulation matrix eigenvalue equation without imposing boundary conditions across the subdomain boundaries can be written as

$$
\begin{aligned}
& {\left[\begin{array}{rrrr}
\overline{\bar{A}}_{1} & \mathbf{0} & \mathbf{0} & \mathbf{0} \\
\mathbf{0} & \overline{\bar{A}}_{2} & \mathbf{0} & \mathbf{0} \\
\mathbf{0} & \mathbf{0} & \ddots & \mathbf{0} \\
\mathbf{0} & \mathbf{0} & \mathbf{0} & \overline{\bar{A}}_{p}
\end{array}\right]_{[2 p(M+1)(N+1)] \times[2 p(M+1)(N+1)]}} \\
& {\left[\begin{array}{r}
\bar{H}_{1} \\
\bar{H}_{2} \\
\vdots \\
\bar{H}_{p}
\end{array}\right]_{[2 p(M+1)(N+1)] \times 1}=\beta^{2}\left[\begin{array}{r}
\bar{H}_{1} \\
\vdots \\
\bar{H}_{p}
\end{array}\right]_{[2 p(M+1)(N+1)] \times 1}}
\end{aligned}
$$

where the subscript of $\overline{\bar{A}}_{i}(i=1,2, \ldots, p)$ represents the numbering of each subdomain. The adjacent subdomains are then connected by imposing the boundary conditions on those matrix elements in (27) that correspond to the boundary nodes of the subdomains. As in [14], we have carefully applied different types of boundary conditions (DBCs or NBCs) to the adjacent subdomains to guarantee numerical stability in our numerical

$$
\begin{array}{rl}
\overline{\bar{A}} & =\left[\begin{array}{cc}
\overline{\bar{D}}_{x 00} \overline{\bar{D}}_{x 00}+\overline{\bar{D}}_{y 00} \overline{\bar{D}}_{y 00}+k_{0}^{2} n^{2} \overline{\bar{I}} & \overline{\bar{D}}_{x 00} \overline{\bar{D}}_{x 00}+\overline{\bar{D}}_{y 00} \overline{\bar{D}}_{y 00}+k_{0}^{2} n^{2} \overline{\bar{I}}
\end{array}\right]_{2 k \times 2 k} \\
\mathbf{0} & \mathbf{0} \\
& =\left[\begin{array}{cc}
\overline{\bar{D}}_{x 00}^{2}+\overline{\bar{D}}_{y 00}^{2}+k_{0}^{2} n^{2} \overline{\bar{I}} & \\
\mathbf{0} & \overline{\bar{D}}_{x 00}^{2}+\overline{\bar{D}}_{y 00}^{2}+k_{0}^{2} n^{2} \overline{\bar{I}}
\end{array}\right]_{2 k \times 2 k .}
\end{array}
$$




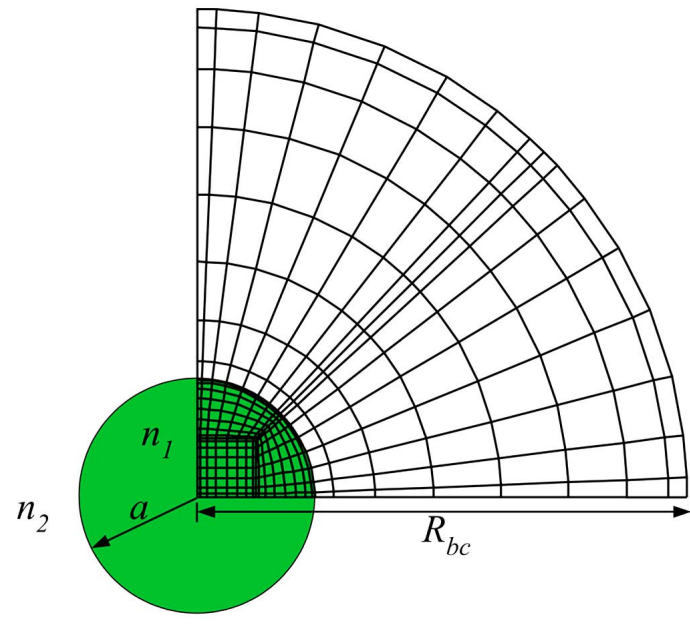

Fig. 3. Cross section of a circular optical fiber with radius $a$ along with the mesh and domain division profile of the computational domain.

scheme. How (27) will be modified when DBCs and NBCs are imposed at the interface between adjacent subdomains can be referred to [14, Appendix]. Finally, the mode propagation constants (eigenvalues) and the corresponding magnetic field distributions (eigenvectors) can be obtained by using the SIPM.

\section{NUMERICAL EXAMPLES AND DISCUSSION}

\section{A. Optical Fiber: Assessment of the PSFD-MS}

We first examine the performance of the proposed PSFD-MS by analyzing a step-index circular optical fiber with core radius $a=0.6 \mu \mathrm{m}$ and a large refractive-index difference with core-index $n_{1}=\sqrt{8}$ and cladding index $n_{2}=1.0$ at $\lambda=$ $1.5 \mu \mathrm{m}$, for which the exact effective index up to 14 digits after the decimal point is found to be $n_{\mathrm{eff}}=2.68401932160108$, where the effective index is defined as $n_{\text {eff }}=\beta / k_{0}$. Fig. 3 shows the typical domain and mesh division profile for the Legendre method, where five subdomains are adopted and the mesh pattern of each subdomain shown is for $M=N=8$. Note that due to the geometrical symmetry, only a quarter of the waveguide cross section needs to be considered. The mesh division profile in each subdomain for the Chebyshev method would be slightly different from that for Legendre method. For each subdomain to be a homogeneous region, the dielectric interfaces should fall on the boundaries of subdomains. Therefore, not all subdomains are of rectangular shape and proper curvilinear mapping technique as discussed in Section IV has been utilized for transformation between the rectangular coordinate and the curved one of a general subdomain. The radius of the computational-domain boundary where we force the total field to be zero (zero boundary condition) is taken to be $R_{b c}=2.5 \mu \mathrm{m}$. We have found that replacing this zero boundary condition with either the perfect electric conductor (PEC) or the perfect magnetic conductor (PMC) would have no difference in the following results. The other two horizontal and vertical linear boundaries of the computational domain are taken to be either PEC or PMC depending on the symmetry properties of the mode to be solved.

Table I lists the calculated effective indexes of the fundamental $\mathrm{HE}_{11}$ mode from both the Legendre and Chebyshev methods for different degrees $(M=N)$ and corresponding numbers of unknowns, or $2 p(M+1)(N+1)$ in (27) with $M=N$ assumed. We see the agreement with the exact effective index up to 11 digits after the decimal point when the degree is up to 22 in both methods. Fig. 4(a) shows the relative errors in the calculated effective indexes of Table I relative to the exact value, i.e., $\left|\left(n_{\text {eff, calculated }}-n_{\text {eff, exact }}\right) / n_{\text {eff, exact }}\right|$, with respect to the number of unknowns for both methods. The same relative errors are plotted in Fig. 4(b) versus the degree $(M=N)$ of the method up to $M=N=30$. Fig. 4 clearly demonstrates the spectral convergence behavior with the relative error dropping to on the order of $10^{-12}$. For degrees greater than 22 , the numerical accuracy can no longer be improved due to the limiting

$$
\begin{aligned}
& \stackrel{\bar{D}}{\xi x}_{\xi}=\left[\begin{array}{ccc}
\overline{\bar{D}}_{\xi x} & \mathbf{0} & \mathbf{0} \\
\mathbf{0} & \ddots & \mathbf{0} \\
\mathbf{0} & \mathbf{0} & \overline{\bar{D}}_{\xi x}
\end{array}\right]_{k \times k} \\
& \stackrel{\bar{D}}{\xi y}_{\xi y}=\left[\begin{array}{ccc}
\overline{\bar{D}}_{\xi y} & \mathbf{0} & \mathbf{0} \\
\mathbf{0} & \ddots & \mathbf{0} \\
\mathbf{0} & \mathbf{0} & \overline{\bar{D}}_{\xi y}
\end{array}\right]_{k \times k} \\
& \widetilde{\bar{D}}_{\eta x}=\left[\begin{array}{cccc}
\operatorname{tr}\left[D_{\eta x(0,0)}\right]_{(N+1) \times(N+1)} & \operatorname{tr}\left[D_{\eta x(0,1)}\right]_{(N+1) \times(N+1)} & \ldots & \operatorname{tr}\left[D_{\eta x(0, N)}\right]_{(N+1) \times(N+1)} \\
\operatorname{tr}\left[D_{\eta x(1,0)}\right]_{(N+1) \times(N+1)} & \operatorname{tr}\left[D_{\eta x(1,1)}\right]_{(N+1) \times(N+1)} & \ldots & \operatorname{tr}\left[D_{\eta x(1, N)}\right]_{(N+1) \times(N+1)} \\
\vdots & \vdots & \vdots & \vdots \\
\operatorname{tr}\left[D_{\eta x(N, 0)}\right]_{(N+1) \times(N+1)} & \operatorname{tr}\left[D_{\eta x(N, 1)}\right]_{(N+1) \times(N+1)} & \ldots & \operatorname{tr}\left[D_{\eta x(N, N)}\right]_{(N+1) \times(N+1)}
\end{array}\right]_{k \times k} \\
& \widetilde{\bar{D}}_{\eta y}=\left[\begin{array}{cccc}
\operatorname{tr}\left[D_{\eta y(0,0)}\right]_{(N+1) \times(N+1)} & \operatorname{tr}\left[D_{\eta y(0,1)}\right]_{(N+1) \times(N+1)} & \ldots & \operatorname{tr}\left[D_{\eta y(0, M)}\right]_{(N+1) \times(N+1)} \\
\operatorname{tr}\left[D_{\eta y(1,0)}\right]_{(N+1) \times(N+1)} & \operatorname{tr}\left[D_{\eta y(1,1)}\right]_{(N+1) \times(N+1)} & \ldots & \operatorname{tr}\left[D_{\eta y(1, M)}\right]_{(N+1) \times(N+1)} \\
\vdots & \vdots & \vdots & \vdots \\
\operatorname{tr}\left[D_{\eta y(M, 0)}\right]_{(N+1) \times(N+1)} & \operatorname{tr}\left[D_{\eta y(M, 1)}\right]_{(N+1) \times(N+1)} & \ldots & \operatorname{tr}\left[D_{\eta y(M, M)}\right]_{(N+1) \times(N+1)}
\end{array}\right]_{k \times k}
\end{aligned}
$$


TABLE I

CONVERgEnCE CHARACTERISTICS OF THE EFFeCTIVE INDEX OF THE FIBER $\mathrm{HE}_{11}$ Mode Calculated by the PSFD-MS With the Degree $(M=N)$ AND THE CORRESPONDING NUMBER OF UNKNOWNS

\begin{tabular}{cccc}
\hline & & \multicolumn{2}{c}{$n_{\text {eff }}($ PSFD $)$} \\
\cline { 3 - 4 }$N$ & Unknowns & Legendre & Chebyshev \\
\hline 8 & 640 & 2.68425785982235 & 2.68448752730184 \\
10 & 1000 & 2.68404624218251 & 2.68408412792738 \\
12 & 1440 & 2.68402183077681 & 2.68402627781399 \\
14 & 1960 & 2.68401951702626 & 2.68401992339789 \\
16 & 2560 & 2.68401933446231 & 2.68401936474090 \\
18 & 3240 & 2.68401932230318 & 2.68401932412803 \\
20 & 4000 & 2.68401932163321 & 2.68401932170647 \\
22 & 4840 & 2.68401932160825 & 2.68401932160645 \\
24 & 5760 & 2.68401932160872 & 2.68401932160792 \\
26 & 6760 & 2.68401932160928 & 2.68401932160907 \\
28 & 7840 & 2.68401932160924 & 2.68401932160890 \\
30 & 9000 & 2.68401932160906 & 2.68401932160913 \\
\hline
\end{tabular}

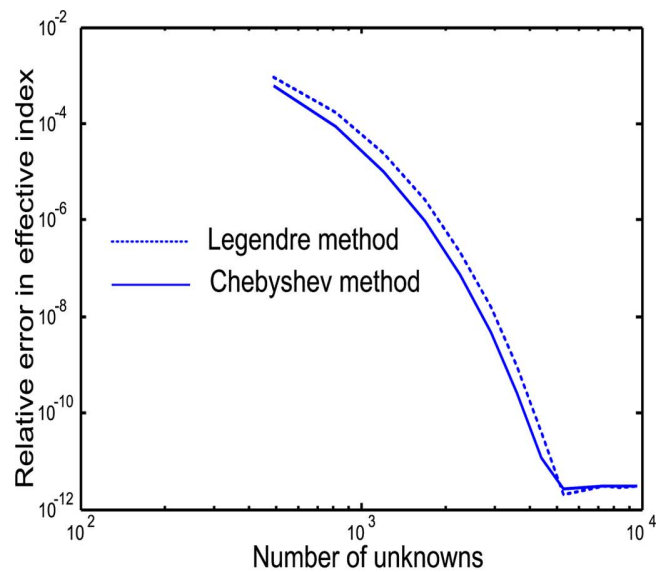

(a)

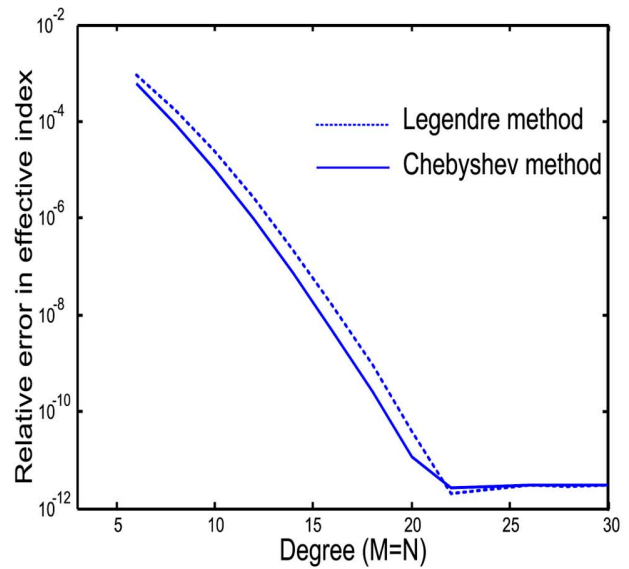

(b)

Fig. 4. Relative error in the effective index of the $\mathrm{HE}_{11}$ mode of the optical fiber as a function of (a) the number of unknowns and (b) the degree $M=N$ of the method.

factors such as truncation errors, possible numerical errors in the transfinite blending function schemes, etc. Since the result based on the Chebyshev method has better accuracy than that

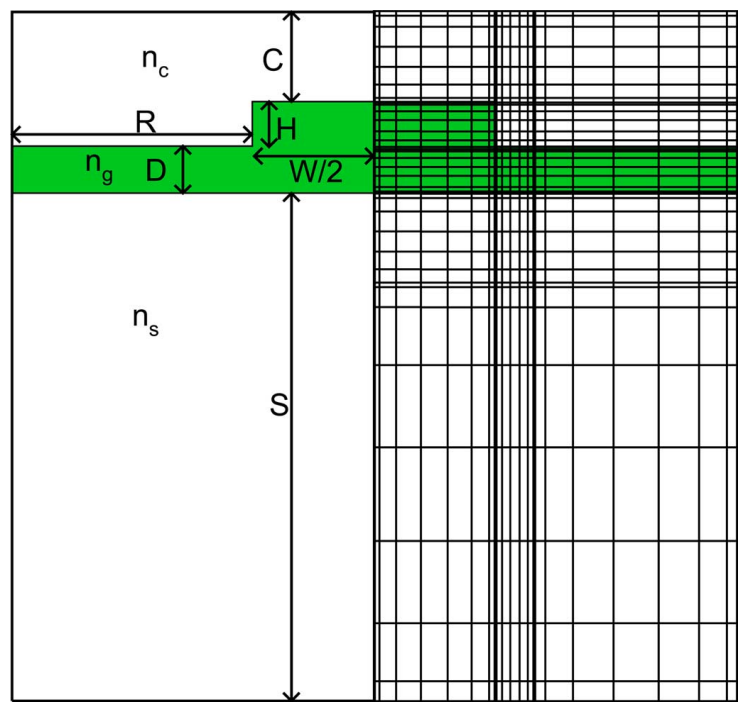

Fig. 5. Cross section of the classical rib waveguide along with the mesh and domain division profile of the computational domain.

TABLE II

CONVERGENCE CHARACTERISTICS OF THE EFFECTIVE INDEX OF THE $\mathrm{H}_{11}^{y}$ Mode OF THE RiB WaVEguide OF Fig. 5 With $D=0.5 \mu \mathrm{m}$ CALCULATED BY THE PSFD-MS

\begin{tabular}{ccc}
\hline$N$ & Unknowns & $n_{\text {eff }}($ PSFD $)$ \\
\hline 6 & 1470 & 3.4133790030 \\
9 & 3000 & 3.4131489900 \\
11 & 4320 & 3.4131357766 \\
13 & 5880 & 3.4131337513 \\
15 & 7680 & 3.4131332450 \\
17 & 9720 & 3.4131329949 \\
18 & 10830 & 3.4131329350 \\
\hline
\end{tabular}

based on the Legendre method, we will adopt the Chebyshev method in the following cases.

Some information of the computer execution time is provided here. Using a personal computer with the Intel Dual Core 4300 1.8-GHz CPU, the calculation with degree 14 takes $7 \mathrm{~s}$ and the effective index agrees with the exact value up to the sixth digit after the decimal point, referring to Table I. With degree 20, it takes $52 \mathrm{~s}$ and the agreement with the exact value becomes up to the tenth digit. It is seen that for the PSFD calculation, the accuracy up to the sixth digit is easily achieved with seconds in personal computers. Such accuracy might not even be obtainable using a simple finite-difference algorithm due to saturation in numerical convergence.

\section{B. Rib Waveguide: A Classical Benchmark Problem}

We then use the Chebyshev PSFD-MS to analyze the rib waveguide with the cross section as shown in Fig. 5 along with the domain and mesh division profile. We only need to consider half of the cross section due to the symmetry of the mode field. The computational domain is arranged to have 15 subdomains and the mesh pattern of each subdomain shown is for $M=N=7$. This waveguide structure has long been a benchmark problem [2], [15]. We assume the same parameters as in [2] and [15]: the rib width $W=3.0 \mu \mathrm{m}, H+D=1.0 \mu \mathrm{m}$ with $D$ varying from 0 to $0.7 \mu \mathrm{m}$, the refractive indexes of 
TABLE III

Calculated Effective indeXes and Normalized Propagation Constants of the Hi1 Mode of the Rib WaVeguide of Fig. 5 From [2], [15] AND THE DEGREE-17 PSFD-MS

\begin{tabular}{cccccc}
\hline$D(\mu \mathrm{m})$ & $n_{\text {eff }}[2]$ & $n_{\text {eff }}$ (PSFD) & $\beta[2]$ & $\beta($ PSFD $)$ & $\beta[15]$ \\
\hline 0.0 & $3.412022 \pm 2 \times 10^{-6}$ & 3.41202174 & 0.2993206 & 0.2993141 & - \\
0.1 & $3.412126 \pm 2 \times 10^{-6}$ & 3.41212624 & 0.3019146 & 0.3019208 & 0.3019 \\
0.2 & $3.412279 \pm 2 \times 10^{-6}$ & 3.41227932 & 0.3057308 & 0.3057390 & - \\
0.3 & $3.412492 \pm 2 \times 10^{-6}$ & 3.41249272 & 0.3110440 & 0.3110621 & 0.3110 \\
0.4 & $3.412774 \pm 2 \times 10^{-6}$ & 3.41277513 & 0.3180788 & 0.3181072 & - \\
0.5 & $3.413132 \pm 3 \times 10^{-6}$ & 3.41313299 & 0.3270104 & 0.3270352 & 0.3270 \\
0.6 & $3.413571 \pm 3 \times 10^{-6}$ & 3.41357166 & 0.3379640 & 0.3379807 & - \\
0.7 & $3.414100 \pm 3 \times 10^{-6}$ & 3.41409997 & 0.3511652 & 0.3511645 & 0.3512 \\
\hline
\end{tabular}

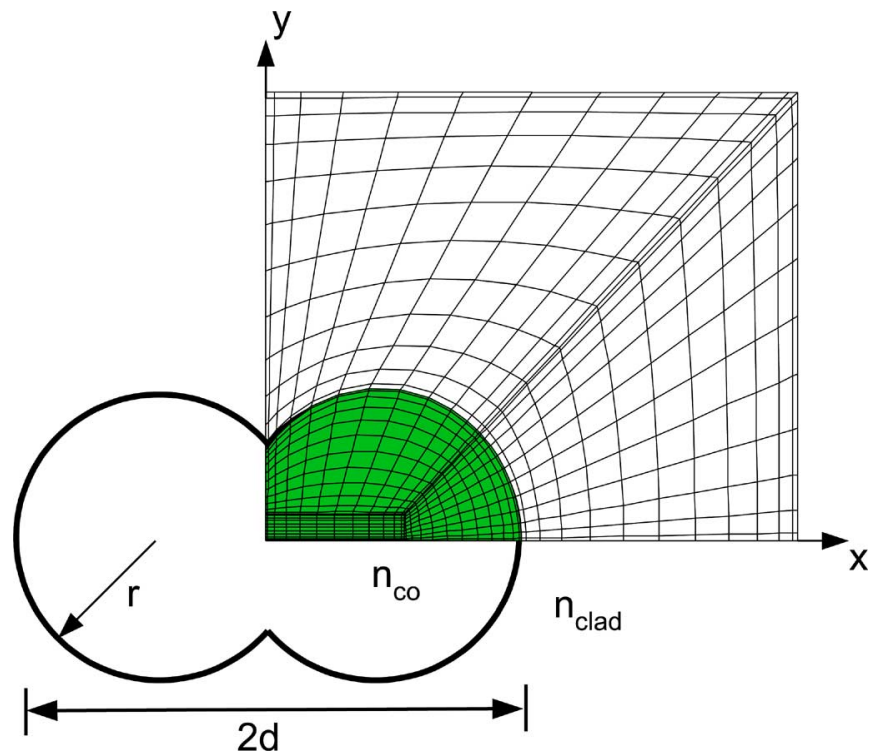

Fig. 6. Cross section of the fused fiber coupler along with the mesh and domain division profile of the computational domain.

the cover $n_{c}$, the guiding layer $n_{g}$, and the substrate $n_{s}$ being $1.0,3.44$, and 3.4 , respectively, and the operating wavelength $\lambda=1.15 \mu \mathrm{m}$. We take the parameters for the computational domain to be $R=3.0 \mu \mathrm{m}, C=1.0 \mu \mathrm{m}$, and $S=5.0 \mu \mathrm{m}$ and assume zero boundary condition at the three outer boundaries.

First, by taking $D=0.5 \mu \mathrm{m}$, we compute the effective index of the $\mathrm{H}_{11}^{y}$ mode for different degrees $(M=N)$ and the results are listed in Table II along with the corresponding numbers of unknowns. It is observed that we have converged seventh digit after the decimal point when $M=N=17$ is employed. Then, we compare our degree-17 results for different values of $D$ with those obtained in [2] using a full-vector waveguide solution algorithm based on high-accuracy finite-difference equations derived for fields satisfying correct boundary conditions at the dielectric corner, as shown in Table III. It is seen that our results confirm the $10^{-6}$ accuracy in the effective index claimed in [2]. Please note that the calculation in [2] involved $152 \times 140$ uniform grids and our degree-17 calculation is with 4860 grids (half the number of unknowns). Also listed in Table III are the corresponding values of the normalized propagation constant defined as $\bar{\beta}=\left(n_{\text {eff }}^{2}-n_{s}^{2}\right) /\left(n_{g}^{2}-n_{s}^{2}\right)$ for comparison with those given in [15] obtained using the modal transverse resonance method (MTRM) [15], [16]. The four-digit values for $\bar{\beta}$ from the MTRM were believed to be exact as stated in [15].
TABLE IV

Calculated Normalized Propagation Constants of the $\mathrm{H}_{11}^{x}$ Mode OF THE RIB WAVEGUIDE OF FIG. 5 From [15] AND THE PSFD-MS USING DEGREES 9 AND 17

\begin{tabular}{cccc}
\hline$D(\mu \mathrm{m})$ & $\beta[15]$ & $\bar{\beta}(\mathrm{PSFD}, N=9)$ & $\bar{\beta}(\mathrm{PSFD}, N=17)$ \\
\hline 0.0 & - & 0.2653237 & 0.2652745 \\
0.1 & 0.2674 & 0.2674715 & 0.2674483 \\
0.2 & - & 0.2706553 & 0.2706354 \\
0.3 & 0.2751 & 0.2751511 & 0.2751333 \\
0.4 & - & 0.2812039 & 0.2811860 \\
0.5 & 0.2890 & 0.2890110 & 0.2889903 \\
0.6 & - & 0.2987571 & 0.2987316 \\
0.7 & 0.3107 & 0.3107102 & 0.3106792 \\
\hline
\end{tabular}

It is seen that the agreement between [2] and this work is up to the fourth digit after the decimal point in $\bar{\beta}$ except for the $D=0.4 \mu \mathrm{m}$ case for which, however, the difference is only smaller than $3 \times 10^{-5}$. The results of [15] appear to have $10^{-4}$ difference for $D=0.7 \mu \mathrm{m}$. Finally, we present in Table IV our $\bar{\beta}$ results for the $\mathrm{H}_{11}^{x}$ mode for degree- 9 and degree-17 calculations and those from [15]. Such results were not given in [2]. It is seen that the four-digit values of [15] agree with our degree-9 results. However, the fourth digit may vary a little in the degree-17 calculation.

\section{Fused Fiber Coupler: Robust Form Birefringence Calculation}

The fused coupler made of two optical fibers [17] is an important device in optical systems, and through elaborate design, it can be manufactured as a power splitter, a polarization beamsplitter, and other devices [18]. The polarization-dependent functions of the coupler can involve beating among the first four vector modes: the lowest order even and odd modes of two orthogonal polarizations. The full-vector modal characteristics, including the propagation constants, the coupling coefficients, and the form birefringence, of the fused coupler have been carefully studied by employing the surface integral equation method (SIEM) [19] and more recently by the improved finite-difference mode solver [3]. Here we demonstrate the efficiency of the PSFD-MS in the analysis of such coupler device. The cross section of the fused coupler is as shown in Fig. 6, again along with the domain and mesh division profile. The computational domain requires only one quarter of the dumbbell-shaped cross section, which is arranged to have five subdomains. The material and geometrical parameters considered, as those used in [3] and [19], are as follows: the core index $n_{\mathrm{co}}=1.45$, the cladding index $n_{\text {clad }}=1$, and the aspect ratio $2 d / 2 r=1.8$, where $r$ 
TABLE V

Convergence Characteristics of the Propagation Constants of the First Four Modes of the Fused Fiber Coupler of Fig. 6 CALCulated By THE PSFD-MS

\begin{tabular}{cccccc}
\hline$N$ & Unknowns & $\beta_{\text {even }}^{x}\left(\mu \mathrm{m}^{-1}\right)$ & $\beta_{\text {even }}^{y}\left(\mu \mathrm{m}^{-1}\right)$ & $\beta_{\text {odd }}^{x}\left(\mu \mathrm{m}^{-1}\right)$ & $\beta_{\text {odd }}^{y}\left(\mu \mathrm{m}^{-1}\right)$ \\
\hline 8 & 810 & 5.979488240 & 5.979444786 & 5.978373503 & 5.978354333 \\
10 & 1210 & 5.979416388 & 5.979386567 & 5.978302901 & 5.978288058 \\
12 & 1690 & 5.979382184 & 5.979359121 & 5.978266694 & 5.978254251 \\
14 & 2250 & 5.979365723 & 5.979346036 & 5.978247616 & 5.978236431 \\
16 & 2890 & 5.979358018 & 5.979339978 & 5.978237588 & 5.978227034 \\
18 & 3610 & 5.979354588 & 5.979337310 & 5.978232419 & 5.978222169 \\
20 & 4000 & 5.979353156 & 5.979336204 & 5.978229827 & 5.978219720 \\
22 & 5290 & 5.979352610 & 5.979335782 & 5.978228566 & 5.978218526 \\
24 & 6250 & 5.979352431 & 5.979335641 & 5.978227973 & 5.978217963 \\
26 & 7290 & 5.979352394 & 5.979335610 & 5.978227702 & 5.978217706 \\
28 & 8410 & 5.979352405 & 5.979335617 & 5.978227583 & 5.978217592 \\
30 & 9610 & 5.979352428 & 5.979335634 & 5.978227531 & 5.978217544 \\
\hline & & & & &
\end{tabular}

TABLE VI

CONVERGENCE CHARACTERISTICS OF THE NORMALIZED COUPLING COEFFICIENTS AND FORM BIREFRINGENCE OF THE FUSED FIBER COUPLER OF FIG. 6 CALCULATED BY THE PSFD-MS

\begin{tabular}{ccccc}
\hline$N$ & Unknowns & $r C_{x}$ & $r C_{y}$ & $B$ \\
\hline 8 & 810 & 0.006433437 & 0.006293286 & 2.6098738 \\
10 & 1210 & 0.006426221 & 0.006339782 & 1.6096498 \\
12 & 1690 & 0.006437782 & 0.006376493 & 1.1413135 \\
14 & 2250 & 0.006452891 & 0.006403821 & 0.9137603 \\
16 & 2890 & 0.006466293 & 0.006423089 & 0.8045461 \\
18 & 3610 & 0.006476329 & 0.006435767 & 0.7553206 \\
20 & 4000 & 0.006483029 & 0.006443523 & 0.7356757 \\
22 & 5290 & 0.006487148 & 0.006447974 & 0.7294754 \\
24 & 6250 & 0.006489541 & 0.006450413 & 0.7286321 \\
26 & 7290 & 0.006490890 & 0.006451716 & 0.7294895 \\
28 & 8410 & 0.006491645 & 0.006452411 & 0.7306091 \\
30 & 9610 & 0.006492074 & 0.006452790 & 0.7315389 \\
\hline
\end{tabular}

is the radius of the individual core. The operating wavelength is $\lambda=1.523 \mu \mathrm{m}$, corresponding to the normalized frequency $V=(2 \pi / \lambda) \cdot r \cdot \sqrt{n_{\mathrm{co}}^{2}-n_{\text {clad }}^{2}}=50$. This is a strongly guiding structure and zero boundary condition is used at the two outer boundaries of the computational domain, which have widths $2.08 d$ and $1.64 d$, respectively.

The lowest order even and odd modes of $x$ and $y$ polarizations have the propagation constants denoted as $\beta_{\text {even }}^{x}, \beta_{\text {even }}^{y}, \beta_{\text {odd }}^{x}$, and $\beta_{\text {odd }}^{y}$, where the even (odd) mode has its main magnetic field component symmetrical (antisymmetrical) about the $y$ axis, and the polarization direction is as usual defined as the main direction of the electric field. The coupling coefficient of $i$ polarization modes, $C_{i}(i=x$ or $y)$, is defined as $C_{i}=\left(\beta_{\text {even }}^{i}-\beta_{\text {odd }}^{i}\right) / 2$ and the form birefringence is defined as $B=\left(r V^{2} / \Delta^{3 / 2}\right)\left(C_{x}-\right.$ $\left.C_{y}\right)$ with $\Delta=\left(n_{\mathrm{co}}^{2}-n_{\mathrm{clad}}^{2}\right) / 2 n_{\mathrm{co}}^{2}$. The coupling coefficient determines the coupling strength of the coupler and the form birefringence plays an important role in the polarization splitting behavior [19]. Since the form birefringence is proportional to $\left(C_{x}-C_{y}\right)$ which is a very small quantity, its accurate caculation has been quite challenging. In [3], which improved the finite-difference mode solution algorithm by carefully treating dielectric interface conditions, nonuniform division scheme was utilized with denser grids near the sharp dielectric corner of the dumbbell-shaped cross section. Similar nonuniform division of the core-cladding boundary near the corner was also employed in the SIEM [19] for improving the efficiency of compu- tation. With a $437 \times 297$ grid mesh, the converged normalized propagation constants of the first four modes obtained in [3] are $\beta_{\text {even }}^{x}=5.97935269 \mu \mathrm{m}^{-1}, \beta_{\text {even }}^{y}=5.97933570 \mu \mathrm{m}^{-1}$, $\beta_{\text {odd }}^{x}=5.97822762 \mu \mathrm{m}^{-1}$, and $\beta_{\text {odd }}^{y}=5.97821760 \mu \mathrm{m}^{-1}$. (Please note that what listed in [3, Table I] should be normalized propagation conatant values.) The corresponding normalized coupling coefficients and the form birefringence are $r C_{x}=$ $0.0064930, r C_{y}=0.0064528$, and $B=0.7491$.

Our PSFD-MS results for different degrees $(M=N)$ and the corresponding numbers of unknowns are shown in Tables $\mathrm{V}$ and VI. For degree 30, the number of unknowns is 9610, and the calculated propagation constants agree with those from [3] given above up to the seventh significant digit. The agreement in $r C_{x}$ and $r C_{y}$ with [3] is up to the third significant digit. As for the form birefringence, we obtain $B=0.7315$, compared with $B=0.7491$ in [3] (and $B=0.7276$ in [19]). Please note that the number of unknowns in [3] has been up to $2 \times 437 \times 297$ but, as mentioned in [3], the form birefringence still does not reach a stable value (which is still decreasing) as the number of divisons increase although the propagation constants converge. However, from Table VI, we are quite confident that the $B$ value is around 0.73 and such close-to-convergence value can be reached with about 5000 unknowns, showing the robustness of the PSFD calculation.

\section{CONCLUSION}

We have proposed and formulated a new full-vectorial optical waveguide mode solution method for waveguides with arbitrary step-index profile based on multidomain pseudospectral methods and name it the PSFD-MS. The method has been described under the $H_{x}-H_{y}$ formulation, in which the Helmholtz equations for the two transverse magnetic field components are solved. The spatial derivatives of the Helmholtz equations are approximated at collocation points by utilizing Legendre-Lagrange (as the Legendre collocation method) or Chebyshev-Lagrange (as the Chebyshev collocation method) interpolating polynomials, and a matrix eigenvalue equation is established with $\beta^{2}$ as the eigenvalue, which is solved for the eigenmodes by the shift inverse power method. Through the multidomain approach and a curvilinear coordinate mapping technique for transforming each curvilinear quadrilateral subdomain into a square one, the computational domain can 
be flexibly divided into suitable number of subdomains to fit general curved interfaces of the refractive-index profile and to facilitate imposing the alternative Dirichlet and Neumann types of boundary conditions across the dielectric interfaces and between adjacent subdomains. Three numerical examples have been presented. The analysis of the fundamental mode of the optical fiber demonstrated the spectral accuracy of the PSFD-MS with the relative error in the effective index as small as on the order of $10^{-12}$. The analysis results for the classical rib waveguide benchmark problem were found to agree excellently with existing high-accuracy ones. And the analysis of the fused fiber coupler showed the robustness of the PSFD-MS in calculating the form birefingence value. The achieved high numerical accuracy is attributed to the inherent high-order scheme of the pseudospectral method with superior convergence behavior due to the specially located collocation points and the rigorous fulfillment of the continuity conditions across the curved dielectric interfaces. Therefore, for a numerical accuracy wanted (the number of accurate digits required), the PSFD-MS will require much less number of unknowns as compared with conventional finite-difference methods, as has been evidenced in the fused coupler example. The PSFD-MS can be easily generalized for treating the leaky waveguide problems by incorporating perfectly matched layer (PML) absorbing boundary conditions [20] into the formulation, as was done in [21] for the finite-difference solver. The preliminary results have recently been reported in [22].

\section{ACKNOWLEDGMENT}

The authors would like to thank the National Center for High-Performance Computing, Hsinchu, Taiwan, R.O.C., for providing useful computing resources.

\section{REFERENCES}

[1] G. R. Hadley, "High-accuracy finite-difference equations for dielectric waveguide analysis I: Uniform regions and dielectric interfaces," J. Lightw. Technol., vol. 20, no. 7, pp. 1210-1218, Jul. 2002.

[2] G. R. Hadley, "High-accuracy finite-difference equations for dielectric waveguide analysis II: Dielectric corners," J. Lightw. Technol., vol. 20, no. 7, pp. 1219-1232, Jul. 2002.

[3] Y. C. Chiang, Y. P. Chiou, and H. C. Chang, "Improved full-vectorial finite-difference mode solver for optical waveguides with step-index profiles," J. Lightw. Technol., vol. 20, no. 8, pp. 1609-1618, Aug. 2002.

[4] M. Koshiba and Y. Tsuji, "Curvilinear hybrid edge/nodal elements with triangular shape for guided-wave problems," J. Lightw. Technol., vol. 18, no. 5, pp. 737-743, May 2000.

[5] P. J. Chiang, C. S. Yang, C. L. Wu, C. H. Teng, and H. C. Chang, "Application of pseudospectral methods to optical waveguide mode solvers," in Proc. OSA 2005 Integrated Photonics Research and Applications (IPRA '05) Technical Digest, 2005, IMG4.

[6] C. Canuto, M. Y. Hussani, A. Quarteroni, and T. Zang, Spectral Methods in Fluid Dynamics. New York: Springer-Verlag, 1988.

[7] B. Yang, D. Gottlieb, and J. S. Hesthaven, "Spectral simulations of electromagnetic wave scattering," J. Comput. Phys., vol. 134, pp. 216-230, 1997.

[8] B. Yang and J. S. Hesthaven, "A pseudospectral method for time-domain computation of electromagnetic scattering by bodies of revolution," IEEE Trans. Antennas Propagat., vol. 47, no. 1, pp. 132-141, Jul. 1999.
[9] J. S. Hesthaven, P. G. Dinesen, and J. P. Lynov, "Spectral collocation time-domain modeling of diffractive optical elements," J. Comput. Phys., vol. 155, pp. 287-306, 1999.

[10] G. Zhao and Q. H. Liu, "The 3-D multidomain pseudospectral timedomain algorithm for inhomogeneous conductive media," IEEE Trans. Antennas Propagat., vol. 52, no. 3, pp. 742-749, Mar. 2004.

[11] Q. H. Liu, "A pseudospectral frequency-domain (PSFD) method for computational electromagnetics," IEEE Antennas Wireless Propagat. Lett., vol. 1, pp. 131-134, 2002.

[12] W. J. Gordon and C. A. Hall, "Transfinite element methods: Blendingfunction interpolation over arbitrary curved element domains," Numer. Math., vol. 21, pp. 109-129, 1973.

[13] C. C. Huang, C. C. Huang, and J. Y. Yang, "A full-vectorial pseudospectral modal analysis of dielectric optical waveguides with stepped refractive index profiles," IEEE J. Sel. Top. Quantum Electron., vol. 11, no. 2, pp. 457-465, Mar./Apr. 2005.

[14] P. J. Chiang, C. P. Yu, and H. C. Chang, "Analysis of two-dimensional photonic crystals using a multidomain pseudospectral method," Phys. Rev. E, vol. 75, p. 026703, Feb. 2007.

[15] C. Vassallo, "1993-1995 optical mode solvers," Opt. Quantum Electron., vol. 29, pp. 95-114, 1997.

[16] A. S. Sudbø, "Numerically stable formulation of the transverse resonance method for vector mode-field calculations in dielctric waveguides," IEEE Photon. Technol. Lett., vol. 5, no. 3, pp. 342-344, Mar. 1993.

[17] B. S. Kawasaki, K. O. Hill, and R. G. Lamont, "Biconical-taper singlemode fiber coupler," Opt. Lett., vol. 6, pp. 327-328, 1981.

[18] M. Eisenmann and E. Weidel, "Single-mode fused biconical coupler optimized for polarization beamsplitting," J. Lightw. Technol., vol. 9, no. 7, pp. 853-858, Jul. 1991.

[19] S.-W. Yang and H.-C. Chang, "Numerical modeling of weakly fused fiber-optic polarization beamsplitters-Part I: Accurate calculation of coupling coefficients and form birefringence," J. Lightw. Technol., vol. 16, no. 4, pp. 685-690, Apr. 1998.

[20] J. P. Berenger, "A perfectly matched layer for the absorption of electromagnetic waves," J. Comput. Phys., vol. 114, pp. 185-200, 1994.

[21] C. P. Yu and H. C. Chang, "Yee-mesh-based finite difference eigenmode solver with PML absorbing boundary conditions for optical waveguides and photonic crystal fibers," Opt. Exp., vol. 12, pp. 6165-6177, 2004.

[22] P. J. Chiang and H. C. Chang, "Analysis of leaky optical waveguides using pseudospectral methods," in Proc. OSA 2006 IPRA Tech. Dig., 2006, ITuA3.

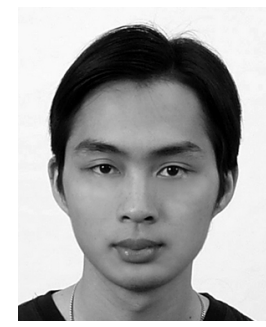

Po-Jui Chiang was born in Kaohsiung, Taiwan, R.O.C., on November 26, 1974. He received the B.S. degree in electronics engineering from National Taiwan University of Science and Technology, Taipei, Taiwan, R.O.C., in 1998 and the M.S. and Ph.D. degrees in electro-optical engineering from National Taiwan University, Taipei, Taiwan, R.O.C., in 2000 and 2007 , respectively.

In February 2007, he joined the faculty of the Electrical Engineering Department, I-Shou University, Kaohsiung County, Taiwan, R.O.C., where he is currently an Assistant Professor. His research interests include numerical methods for solving optical waveguide problems.

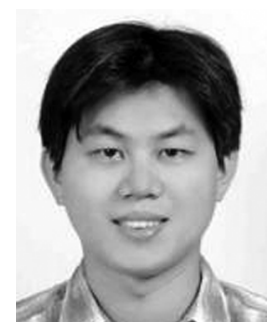

Chin-Lung Wu was born in Tainan, Taiwan, R.O.C., on August 10, 1976. He received the B.S. degree in electronics engineering from the National Taiwan University of Science and Technology, Taipei, Taiwan, R.O.C., in 1998 and the M.S. degree in communication engineering from National Taiwan University, Taipei, in 2003.

He is currently with Himax Analogic, Inc., Taipei, Taiwan, R.O.C., as an integrated circuit Design Engineer. 


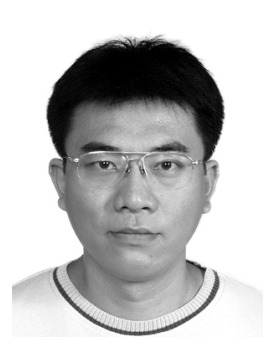

Chun-Hao Teng was born in Tainan, Taiwan, R.O.C., on February 14, 1970. He received the diploma in mechanical engineering from National Taipei Institute of Technology, Taipei, Taiwan, R.O.C., in 1990, and the M.S. degree in mechanical engineering from Clarkson University, Potsdam, NY, in 1996, and the M.S. and Ph.D. degrees in applied mathematics from Brown University, Providence, RI, in 2001.

$\mathrm{He}$ is currently an Assistant Professor in the Department of Mathematics at National Cheng Kung University, Tainan, Taiwan, R.O.C. His research interests are the developments and applications of high-order numerical methods for partial differential equations.

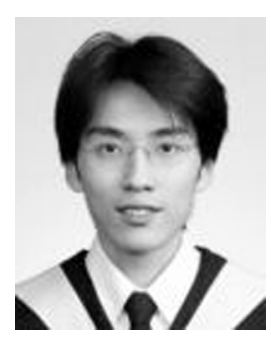

Chu-Sheng Yang was born in Taipei, Taiwan, R.O.C., on November 1, 1980. He received the B.S. degree in electrical engineering from National Taiwan University, Taipei, Taiwan, R.O.C., in 2002. He is currently a graduate student in the Department of Electrical Engineering and Computer Science, University of Michigan, Ann Arbor.

During 2002-2004, he conducted research on the problems of optical switching and bistability, optical tristability, and nonlinear periodic structures in the Computational Photonics and Electromagnetic Laboratory, National Taiwan University.

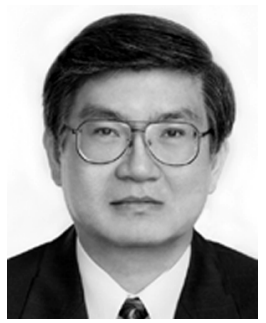

Hung-chun Chang (S'78-M'83-SM'00) was born in Taipei, Taiwan, R.O.C., on February 8, 1954. He received the B.S. degree from National Taiwan University, Taipei, R.O.C., in 1976, and the M.S. and $\mathrm{Ph} . \mathrm{D}$. degrees from Stanford University, Stanford, CA, in 1980 and 1983, respectively, all in electrical engineering.

From 1978 to 1984 , he was with the Space, Telecommunications, and Radioscience Laboratory of Stanford University. In August 1984, he joined the faculty of the Electrical Engineering Department of National Taiwan University, where he is currently a Distinguished Professor. He served as Vice-Chairman of the Electrical Engineering Department from 1989 to 1991, and Chairman of the newly established Graduate Institute of Electro-Optical Engineering from 1992 to 1998. His current research interests include the electromagnetic theory, design, and application of photonic structures and devices for fiber optics, integrated optics, optoelectronics, nanophotonics, and plasmonics.

Dr. Chang is a member of Sigma Xi, the Phi Tau Phi Scholastic Honor Society, the Chinese Institute of Engineers, the Photonics Society of ChineseAmericans, the Optical Society of America, the Electromagnetics Academy, and China/SRS (Taipei) National Committee (a Standing Committee member during 1988-1993 and since 2006, and the Commission B Official Member since 2002) of the International Union of Radio Science (URSI). He served as the IEICE (Japan) Overseas Area Representative in Taipei from 2002 to 2007. In 1987, he was among the recipients of the Young Scientists Award at the URSI XXIInd General Assembly. In 1993, he was one of the recipients of the Distinguished Teaching Award sponsored by the R.O.C. Ministry of Education. In 2004, he received the Merit NSC Research Fellow Award sponsored by the National Science Council, R.O.C. 